\title{
Provision of dental health services during the nationwide COVID-19 lockdown in Bhutan
}

\author{
Tashi Dema ${ }^{1}$, Dawa Gyeltshen ${ }^{1}$
}

AFFILIATION

1 Eusa Hospital, Ministry of Health, Wangdue Phodrang, Bhutan

CORRESPONDENCE TO

Tashi Dema. Eusa Hospital, Ministry of Health, Wangdue Phodrang, Bhutan. E-mail: tashidgyaltsho504@gmail.com ORCID ID: https://orcid. org/0000-0002-9289-9265

Popul. Med. 2021;3(December):34
KEYWORDS

Bhutan, COVID-19, dental health services, oral health worker, lockdown

Received: 10 November 2021, Accepted: 6 December 2021

https://doi.org/10.18332/popmed/144621

\section{Dear Editor,}

Bhutan responded to the COVID-19 pandemic by implementing various public health measures including nationwide lockdown on two separate occasions, and vaccination $^{1}$. These responses hindered the provision of healthcare services in Bhutan including dental health services. Oral health workers (dentists, dental specialists, dental hygienists, dental technicians) are among the highest occupational risk categories for the transmission and contraction of COVID-19. Routine dental treatments include the use of a turbine handpiece, air-water syringes, and ultrasonic scalers that produce significant amounts of aerosols considered to be a high risk for the spread of the virus $^{2}$. Although the dental healthcare services were not included in the essential healthcare service list, the Ministry of Health, Bhutan, followed the guidelines of the World Health Organization (WHO), the American Dental Association (ADA), the National Health Service of the United Kingdom and the National Health Commission of China, along with other dental associations worldwide to formulate a protocol to provide dental healthcare services safely.

The elective dental procedures were postponed whilst only emergency dental treatments were provided during the lockdown periods. However, in order to ensure universal health coverage, even during the periods of lockdown, people seeking dental treatment were covered through teleconsultation $^{3}$. After teleconsultation, they were screened as basic, non-emergency, and emergency dental healthcare seekers ${ }^{3}$. Those patients requiring emergency medications, but not requiring in-hospital treatment, were treated at home by the identified health workers. Only patients requiring in-hospital emergency procedures were asked to visit the hospital $^{3}$. A short message was sent to them which they had to show to the police who were on duty to monitor movement in the urban areas and keep records, whereas in rural areas, local leaders were informed of their movement.
The patients followed COVID-19 protocols such as wearing a facemask, face shield and hand hygiene, when visiting the hospital. On reaching the hospital, they had to undergo a mandatory antigen test. Following a negative report, rinsing of their mouth either with hydrogen peroxide $(0.5-1.0 \%)$ or povidone-iodine $(0.2 \%)$ before the procedure was followed ${ }^{3}$. Oral healthcare workers followed strict protocols and used full personal protective equipment during the procedure ${ }^{3}$.

In addition, dental emergency procedures were also carried out at the homes of the patients for the elderly and patients with disabilities. A team of oral healthcare workers was deployed who followed strict COVID-19 containment protocols. The same COVID-19 protocols were followed by the patients. After the procedure, the information regarding people who consulted for dental treatment were recorded and asked to visit hospitals when the condition improved or the vaccine received ${ }^{3}$. This carefully considered protocol proved successful because no patient requiring dental healthcare services was left out, even during the periods of lockdown. Also, no healthcare worker or patient contracted COVID-19 in this manner.

Considering oral healthcare workers are among the highest occupational risk categories for the transmission and contraction of COVID-19, Bhutan efficiently provided oral health services to the people without placing at risk both the patients and the healthcare givers. Since there is no definite data recorded for how many and what kind of oral health services have been provided during both lockdowns, it would be helpful to carry out surveys in this field to better plan similar events for similar situations in the future. This will be crucial in further refining protocols in the Bhutanese context.

\section{REFERENCES}

1. Gyeltshen D, Dema T, Ahmadi A, Lucero-Prisno DE. Bhutan's COVID-19 Vaccination Efforts and the Evolution of COVID-19 
Cases in the Aftermath. Asia Pac J Public Health. Published online October 21, 2021. doi:10.1177/10105395211052178

2. Statement on the second meeting of the International Health Regulations (2005) Emergency Committee regarding the outbreak of novel coronavirus (2019-nCoV). World Health Organization. January 30, 2020. Accessed November 3, 2021. https://www.who.int/news/item/30-01-2020-statementon-the-second-meeting-of-the-international-healthregulations-(2005)-emergency-committee-regarding-theoutbreak-of-novel-coronavirus-(2019-ncov)

3. Bajgai GP, Ngedup S. RECOMMENDATIONS FOR DENTAL PRACTICE DURING COVID19 PANDEMIC AND LOCKDOWN IN BHUTAN. Journal of Critical Reviews. 2020;7(18):46394643. doi:10.31838/jcr.07.18.579

\section{CONFLICTS OF INTEREST}

The authors have completed and submitted the ICMJE Form for Disclosure of Potential Conflicts of Interest and none was reported.

\section{FUNDING}

There was no source of funding for this research.

\section{DATA AVAILABILITY}

Data sharing is not applicable to this article as no new data were created.

PROVENANCE AND PEER REVIEW

Not commissioned; internally peer reviewed. 